\title{
EFEITO DA SUBSTITUIÇÃo POR ÁTOMOS DE FLÚOR NO EQUILÍBRIO CONFORMACIONAL DE CHALCONA
}

Dari Cesarin-Sobrinho e José Carlos Netto-Ferreira*

Departamento de Química, Universidade Federal Rural do Rio de Janeiro, 23851-970 Seropédica - RJ

Raimundo Braz-Filho

Setor de Química de Produtos Naturais, LCQUI-CCT, Universidade Estadual do Norte Fluminense, 28015-620 Campos - RJ

Recebido em 30/5/00; aceito em 9/2/01

\begin{abstract}
EFFECT OF FLUORINE ATOM SUBSTITUTION ON THE CONFORMATIONAL EQUILIBRIUM OF CHALCONE. Chalcone (1) and its fluorinated derivatives $\mathbf{2 - 4}$, as well as their cyclic analogues 5-10, were synthesized through an aldol condensation reaction between the corresponding ketone and aldehyde. These compounds were characterized by IR, EIMS and ${ }^{1} \mathrm{H}$ and ${ }^{13} \mathrm{C}$ NMR spectral data. Modern NMR techniques allowed us to conclude that the compounds obtained show $E$ configuration. These techniques were also employed to investigate the equilibrium involving the $s$-cis and $s$-trans conformations of 14, with this equilibrium being dependent on the fluorine substitution on both aromatic rings, A or B. IR studies indicated that the yield of the $s$-cis conformation in the fluorinated derivatives is $57.4 \pm 1.4$; $88.1 \pm 0.4$ and $66.4 \pm 0.7 \%$, for $\mathbf{2}, 3$ and 4 , respectively, based on previous ${ }^{1} \mathrm{H}$ NMR calculations for chalcone. Theoretical calculations, using the MMX method, were employed to justify the variation of chemical shifts for the fluorinated derivatives and cyclic analogues. These chemical shifts are consequence of the anisotropic effect showed by the carbonyl group on these compounds.
\end{abstract}

Keywords: chalcone; conformational equilibrium; spectral data.

\section{INTRODUÇÃO}

A rotação em torno das ligações simples carbono-carbono é um processo bem conhecido e largamente estudado em diversas classes de substâncias orgânicas ${ }^{1}$. Podemos destacar, em particular, a rotação livre em torno da ligação simples em sistemas conjugados do tipo $\mathrm{X}=\mathrm{CR} 1-\mathrm{CR} 2=\mathrm{Y}$, onde também é amplamente aceita a hipótese de que, quando possível, esses sistemas adotam conformações planas permitindo que a estrutura seja estabilizada pelo processo de ressonância. São possíveis para esse sistema duas estruturas planas, $s$-cis e $s$-trans, e, em muitos dos casos, pode ser estabelecido um equilíbrio entre estas duas conformações, sendo a proporção $s$-cis/s-trans dependente, exclusivamente, de como os grupos substituintes $\mathrm{X}, \mathrm{Y}, \mathrm{R} 1$ e R2 interagem entre si e com o ambiente químico.

De acordo com a natureza dos substituintes, podemos classificar as interações quanto aos efeitos estéricos causados por substituintes volumosos ${ }^{2-22}$, aos efeitos eletrônicos decorrentes da diferença de eletronegatividade entre os átomos ou grupos substituintes $^{23}$, ou a presença de sítios ácido/base de Lewis possibilitando a formação de ligações de hidrogênio e/ou complexos intra- ${ }^{24} \mathrm{e}$ intermoleculares ${ }^{25}$.

Outro fator muito importante para o estabelecimento da estabilidade conformacional é a polaridade do solvente. Uma particularidade das substâncias carbonílicas e análogas, quando comparadas aos alquenos, é a dependência da população dos confôrmeros com a polaridade do solvente. Esta subordinação torna-se particularmente pronunciada quanto maior for a diferença entre os momentos dipolares de cada confôrmero individualmente ${ }^{26}$.

Esse trabalho tem como objetivo contribuir para o estabelecimento de uma maior compreensão do comportamento molecular em solventes apolares das chalconas fluoradas $\mathbf{2}$ - $\mathbf{4}$, com diferentes graus de substituição de átomos de hidrogênio por átomos de flúor em ambos os anéis aromáticos. Visto que a geometria molecular pode desempenhar um papel preponderante no controle de muitos processos reacionais, especialmente nos processos de fotoisomerização e fotodimerização ${ }^{27}$, será feita uma comparação com os análogos cíclicos $\mathbf{5}$ - 10, que apresentam configuração e conformação definidas.
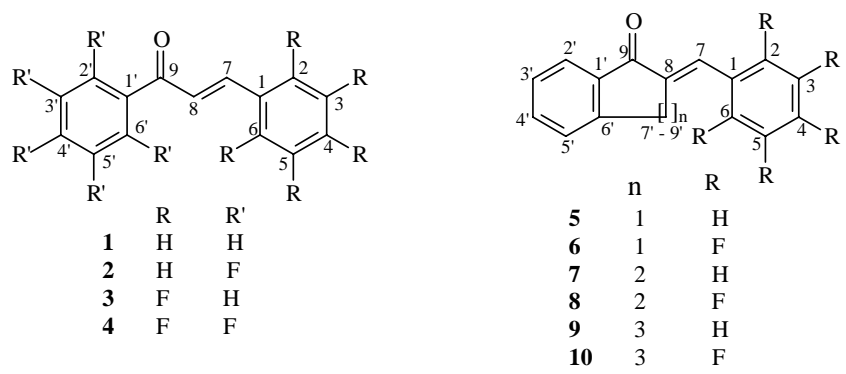

Esquema 1

\section{PARTE EXPERIMENTAL}

Síntese de chalcona (1), das chalconas fluoradas 2-4 e dos análogos cíclicos 5-10

A chalcona (1), os derivados pentafluorados $\mathbf{2}$ e $\mathbf{3}$, o decafluorado 4 e os análogos cíclicos 5-10 foram sintetizados por reação de condensação aldólica em meio básico ${ }^{28}$, através da seguinte metodologia geral. Em um balão de $50 \mathrm{~mL}$, imerso em banho de gelo, colocou-se $10 \mathrm{~mL}$ de $\mathrm{EtOH} / \mathrm{H}_{2} \mathrm{O}$ (6:4), uma solução de $\mathrm{KOH}$ de razão molar 1:10 em relação à concentração molar da cetona $(0,24 \mathrm{M})$ e o aldeído $(0,26 \mathrm{M})$ sob agitação magnética, seguindo-se a adição de metade da massa de cetona. Após ser verificado o início da reação, adicionou-se o restante da cetona. A reação foi mantida sob agitação constante até a obtenção de um precipitado, o qual foi filtrado, pulverizado e lavado com $\mathrm{H}_{2} \mathrm{O}$ destilada até $\mathrm{pH}$ neutro, sendo posteriormente recristalizado sucessivamente em solução de $\mathrm{EtOH} / \mathrm{H}_{2} \mathrm{O}$. O produto obtido foi seco, acondicionado em frasco escuro e mantido em refrigerador, devido à sua foto- e termolabilidade. Os pontos de fusão para 1-10, bem como os dados de espectros de massa (picos correspondentes aos íons moleculares e os decorrentes dos principais processos de fragmentação) utilizados para a confirmação da estrutura do produto obtido nas diversas sínteses, encontram-se listados na Tabela 1. 
Tabela 1. Dados obtidos do espectro de massas e pontos de fusão para chalcona (1), chalconas fluoradas 2-4 e análogos cíclicos 5-10.

\begin{tabular}{|c|c|c|c|c|c|c|c|c|c|c|c|c|c|c|c|c|c|c|c|c|}
\hline & \multicolumn{2}{|c|}{1} & \multicolumn{2}{|c|}{2} & \multicolumn{2}{|c|}{3} & \multicolumn{2}{|c|}{4} & \multicolumn{2}{|c|}{5} & \multicolumn{2}{|c|}{6} & \multicolumn{2}{|c|}{7} & \multicolumn{2}{|c|}{8} & \multicolumn{2}{|c|}{9} & \multicolumn{2}{|c|}{10} \\
\hline & $m / z$ & $(\%)$ & $m / z$ & $(\%)$ & $m / z$ & $(\%)$ & $m / z$ & $(\%)$ & $m / z$ & $(\%)$ & $m / z$ & $(\%)$ & $m / z$ & $(\%)$ & $m / z$ & $(\%)$ & $m / z$ & $(\%)$ & $m / z$ & $(\%)$ \\
\hline $\mathrm{M}^{+\cdot}$ & 208 & 85 & 298 & 60 & 298 & 100 & 388 & 80 & 220 & 67 & 310 & 100 & 234 & 51 & 324 & 100 & 248 & 86 & 338 & 63 \\
\hline M-H & 207 & 100 & 297 & 100 & 297 & 0 & 387 & 0 & 219 & 100 & 309 & 3 & 233 & 100 & 323 & 4 & 247 & 10 & 337 & 0 \\
\hline M-F & - & - & 279 & 2 & 279 & 12 & 369 & 0 & - & - & 291 & 55 & - & - & 305 & 94 & - & - & 319 & 15 \\
\hline $\mathrm{M}-\mathrm{CO}$ & 180 & 6 & 270 & 0 & 270 & 12 & 360 & 30 & 192 & 16 & 282 & 25 & 206 & 2 & 296 & 5 & - & - & - & - \\
\hline $\mathrm{M}-\mathrm{COH}$ & 179 & 24 & 269 & 1 & 269 & 5 & 359 & 0 & 191 & 55 & 281 & 40 & 205 & 5 & 295 & 4 & 219 & 12 & 309 & 4 \\
\hline $\mathrm{PF}{ }^{\circ} \mathrm{C}$ & \multicolumn{2}{|c|}{$56-58^{\mathrm{a}}$} & \multicolumn{2}{|c|}{$92-94$} & \multicolumn{2}{|c|}{$74-76$} & \multicolumn{2}{|c|}{$50-52$} & \multicolumn{2}{|c|}{$112-114^{b}$} & \multicolumn{2}{|c|}{$103-104^{c}$} & \multicolumn{2}{|c|}{ 93-95 } & \multicolumn{2}{|c|}{ óleo } & \multicolumn{2}{|c|}{$72-74$} & \\
\hline
\end{tabular}

${ }^{\mathrm{a}}$ ponto de fusão na literatura ${ }^{29}: 57-58{ }^{\circ} \mathrm{C}$; ${ }^{b}$ ponto de fusão na literatura ${ }^{30}: 113-114{ }^{\circ} \mathrm{C}$; ${ }^{\mathrm{c}}$ ponto de fusão na literatura ${ }^{31}$ : $105{ }^{\circ} \mathrm{C}$; d material apresentando cor levemente violácea, indicando possível degradação, o que impossibilitou a determinação correta de seu ponto de fusão.

\section{Métodos Gerais}

Os pontos de fusão foram determinados num aparelho tipo Kofler e não foram corrigidos.

Os espectros de massas (EM) foram obtidos através de um espectrômetro de massas HP $5995(70 \mathrm{eV})$ acoplado à cromatografia gasosa (EM-CG), empregando uma coluna capilar BP1, de sílica fundida, de $12 \mathrm{~m}$.

Os espectros de RMN uni- [1D: ${ }^{1} \mathrm{H},{ }^{13} \mathrm{C}$ e as experiências de efeito nuclear Overhauser $\left({ }^{1} \mathrm{Hx}{ }^{1} \mathrm{H}-\mathrm{NOE}\right)$ por subtração de espectros] e bidimensionais [2D: correlações homonuclear de hidrogênio $\left({ }^{1} \mathrm{Hx}^{1} \mathrm{H}-\mathrm{COSY}\right)$ e heteronuclear de carbono e hidrogênio (HETCOSY) através de uma ligação $\left({ }^{13} \mathrm{Cx}^{1} \mathrm{H}-\mathrm{COSY}\right.$ $\left.{ }^{1} \mathrm{~J}_{\mathrm{CH}}\right)$ foram obtidos em um aparelho Bruker AC-200 $\left({ }^{1} \mathrm{H}\right.$ : $200 \mathrm{MHz} ;{ }^{13} \mathrm{C}: 50,3 \mathrm{MHZ}$ ), usando-se $\mathrm{CDCl}_{3}$ como solvente e TMS como padrão interno ${ }^{32}$. Os parâmetros de aquisição dos espectros de RMN ${ }^{1} \mathrm{H}$ foram os seguintes: número de espectros (NS) 128; largura do pico (PW) 2,0 $\mu \mathrm{s}$; intervalo entre picos (RD) 1,00 $\mu \mathrm{s}$; tamanho (SI) 16.384 e tempo de aquisição (AQ) $1,425 \mathrm{~s}$. Para a aquisição de espectros de $\mathrm{RMN}{ }^{13} \mathrm{C}$, os seguintes parâmetros foram utilizados: (NS) 30.000; (PW) 6,5 $\mu \mathrm{s}$; (RD) $0,0 \mu \mathrm{s}$; (SI) 16.384 e (AQ) 0,655 s. Foram também obtidos espectros de RMN 1D: ${ }^{1} \mathrm{H}$ para as chalconas $\mathbf{1 - 4} \mathrm{em}$ $\mathrm{CCl}_{4}$, empregando TMS em $\mathrm{CDCl}_{3}$ como referência externa.

Os espectros na região do infravermelho (IV) foram obtidos em espectrofotômetro Perkin-Elmer 1420, utilizando-se $\mathrm{CCl}_{4}$ ou $\mathrm{CHCl}_{3}$ como solvente e cela de $\mathrm{NaCl}$ com caminho ótico de $0,1 \mathrm{~cm}$, e calibrados com filme de poliestireno através da banda em $1601,4 \mathrm{~cm}^{-1}$. A integração das bandas de infravermelho foi realizada pelo método da pesagem da área dos sinais a partir de triplicatas em três concentrações diferentes nas proporções $1: 2: 3$.

Para o cálculo da concentração das conformações por infravermelho, considerou-se que na série de compostos 2-4, derivados fluorados da chalcona (1), as intensidades específicas $\mathrm{i}_{\mathrm{C}=\mathrm{O}}(s-c i s)$ e $\mathrm{i}_{\mathrm{C}=\mathrm{O}}(s$-trans $)$ sejam constantes ${ }^{33,34}$. O fator de conversão para estimar os coeficientes de absorção relativos para as conformações $s$-cis/s-trans foi obtido a partir dos dados apresentados na literatura para a chalcona $(\mathbf{1})^{22}$, juntamente com os respectivos experimentos de IV em solução de $\mathrm{CHCl}_{3}$ e em $\mathrm{CCl}_{4}$.

Os cálculos teóricos foram desenvolvidos através do método da mecânica molecular disponível no programa PC-MODEL (versão 4.0, 1997). Todas as etapas foram realizadas em computador Pentium I $166 \mathrm{Mhz}$, sob o sistema operacional Windows 95. A construção das matrizes correspondentes às estruturas estudadas e os cálculos de mecânica molecular foram feitos com o módulo SYBYLL do programa PC Spartan Plus $1.5^{35}$.

Os dados de $\mathrm{RMN}{ }^{1} \mathrm{H}$ e ${ }^{13} \mathrm{C}$ podem ser encontrados nas Tabelas 2-4 enquanto que os dados de IV encontram-se na Tabela 5 .

\section{RESULTADOS E DISCUSSÃO}

Com base nos resultados fornecidos por espectros de RMN ${ }^{1} \mathrm{H}$ e RMN ${ }^{13} \mathrm{C}(\mathrm{HBBD}=$ Hydrogen Broad Band Decoupled e DEPT $=$ Distortionless Enhancement by Polarization Transfer), uni- e bidimensionais de correlação homonuclear de hidrogênio $\left({ }^{1} \mathrm{Hx}{ }^{1} \mathrm{H}-\mathrm{COSY}\right)$ e heteronuclear (HETCOSY) de hidrogênio e carbono-13 ${ }^{13} \mathrm{C}$ detectado, $\left.{ }^{13} \mathrm{Cx}^{1} \mathrm{H}-\mathrm{COSY}-{ }^{\mathrm{n}} \mathrm{J}_{\mathrm{CH}}, \mathrm{n}=1\right)$, foi possível correlacionar os deslocamentos químicos dos átomos de hidrogênio $\left(\delta_{\mathrm{H}}\right)$ e carbono $\left(\delta_{\mathrm{C}}\right)$ da chalcona $(\mathbf{1})$ e seus derivados fluorados 2-4, bem como dos análogos cíclicos 5-10 (Tabela 2). A análise comparativa dos espectros de RMN ${ }^{13} \mathrm{C}-\mathrm{HBBD}$ e RMN ${ }^{13} \mathrm{C}$-DEPT foi usada para reconhecer os sinais correspondentes aos carbonos quaternários, metínicos e metilênicos.

Uma interpretação correta dos valores de $\delta_{\mathrm{H}}$ e $\delta_{\mathrm{C}}$ para as chalconas 1-4 deve considerar as mudanças na posição do equilíbrio conformacional $s$-cis (1a)/s-trans (1) locamento químico é uma média ponderada dos efeitos sofridos pelo núcleo em relação ao ambiente em que se encontra, então um aumento da concentração do isômero $s$-cis e, conseqüentemente, uma diminuição na concentração de $s$-trans, acarreta alterações significativas nas posições de absorção de ${ }^{1} \mathrm{H}$ e ${ }^{13} \mathrm{C}$.
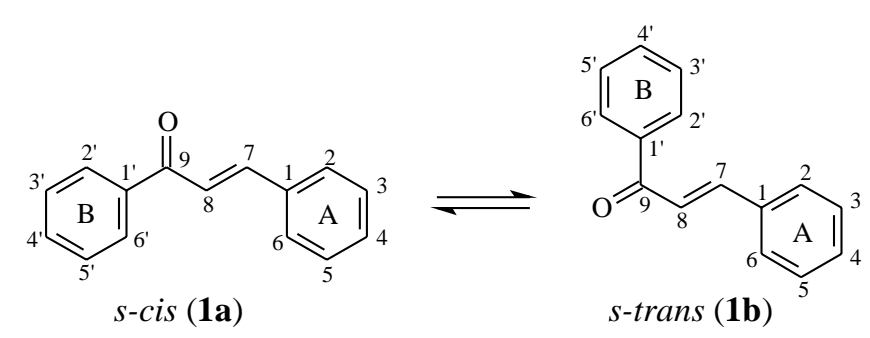

Os núcleos mais importantes a serem analisados no esqueleto chalcônico são o H-7 ( $\beta$ em relação ao grupo carbonila), H8 ( $\alpha$ em relação ao grupo carbonila), C-7, C-8 e o carbono carbonílico C-9, sendo três os efeitos principais que influenciam o deslocamento químico de ${ }^{1} \mathrm{H}$ e ${ }^{13} \mathrm{C}$ : a) o efeito anisotrópico exercido principalmente pelos anéis aromáticos e, em menor intensidade, pela carbonila sobre os hidrogênios $\mathrm{H}-7$ e $\mathrm{H}-8$; b) o efeito de proteção gama $(\gamma)$ exercido principalmente pelo átomo de oxigênio do grupo carbonila nos átomos de carbono C-7 e orto (C-2'/C-6') do anel aromático B, no caso de espectros de $\mathrm{RMN}{ }^{13} \mathrm{C}$; c) os efeitos indutivo e mesomérico transmitidos através da conjugação tanto para os hidrogênios quanto para os carbonos.

Os estudos por RMN realizados para chalcona (1) e seus derivados 2-4 mostraram que os deslocamentos químicos dos hidrogênios olefínicos $\mathrm{H}-7$ e H-8 dependem principalmente do ambiente químico gerado pelos anéis aromáticos e seus substi- 
Tabela 2. Dados de $\mathrm{RMN}{ }^{1} \mathrm{H}$ e ${ }^{13} \mathrm{C}$ para os compostos 1-10. Deslocamentos químicos em $\delta\left(\delta_{\mathrm{C}}\right.$ e $\left.\delta_{\mathrm{H}}\right)$, em CDCl ${ }_{3}$ e TMS como referência interna.

\begin{tabular}{|c|c|c|c|c|c|c|c|c|c|c|}
\hline$\delta_{\mathrm{C}} / \delta_{\mathrm{H}}$ & 1 & 2 & 3 & 4 & 5 & 6 & 7 & 8 & 9 & 10 \\
\hline C-1' & 138,14 & b & $\mathrm{b}$ & b & $\mathrm{b}$ & $\mathrm{b}$ & $\mathrm{b}$ & $\mathrm{b}$ & 138,57 & b \\
\hline C-2' & 128,37 & $\mathrm{a}$ & 128,58 & $\mathrm{a}$ & 124,04 & 124,75 & 127,73 & 127,28 & 129,06 & 129,13 \\
\hline C-3' & 128,37 & $\mathrm{a}$ & 128,79 & $\mathrm{a}$ & 125,92 & 126,18 & 126,58 & 128,47 & 126,94 & 127,12 \\
\hline C-4, & 132,67 & a & 133,43 & $\mathrm{a}$ & 134,99 & 135,44 & 132,87 & 133,88 & 132,34 & 132,91 \\
\hline $\mathrm{C}-5$, & 128,37 & $\mathrm{a}$ & 128,79 & $\mathrm{a}$ & 127,36 & 127,90 & 127,84 & 128,43 & 128,88 & 129,03 \\
\hline C-6' & 128,37 & $\mathrm{~b}$ & 128,58 & $\mathrm{~b}$ & b & b & b & $\mathrm{b}$ & 139,58 & b \\
\hline C-1 & 134,79 & 133,58 & b & $\mathrm{b}$ & $\mathrm{b}$ & $\mathrm{b}$ & $\mathrm{b}$ & $\mathrm{b}$ & 137,73 & $\mathrm{~b}$ \\
\hline$C-2,6$ & 128,85 & 129,09 & $\mathrm{a}$ & $\mathrm{a}$ & 130,45 & $\mathrm{a}$ & 129,49 & $\mathrm{a}$ & 129,39 & $\mathrm{a}$ \\
\hline C- 3,5 & 128,52 & 128,85 & a & $\mathrm{a}$ & 128,65 & $\mathrm{a}$ & 128,06 & a & 128,52 & $\mathrm{a}$ \\
\hline C-4 & 130,45 & 131,67 & $\mathrm{a}$ & $\mathrm{a}$ & 129,43 & $\mathrm{a}$ & 128,18 & $\mathrm{a}$ & 128,56 & $\mathrm{a}$ \\
\hline $\mathrm{C}-7$ & 144,71 & 148,21 & 127,66 & 130,09 & 134,38 & 117,73 & 136,13 & 119,56 & 135,64 & $\mathrm{~b}$ \\
\hline C-8 & 121,99 & 126,06 & 128,79 & 131,95 & $\mathrm{~b}$ & b & $\mathrm{b}$ & b & 137,93 & 121,20 \\
\hline C-9 & 190,40 & 183,70 & 189,22 & 182,86 & 194,04 & 192,50 & 187,24 & 186,11 & 197,98 & 196,13 \\
\hline C-7' & - & - & - & - & 32,11 & 31,70 & 28,37 & - & 31,58 & 31,22 \\
\hline C-8, & - & - & - & - & - & - & 26,75 & - & 26,52 & 25,79 \\
\hline C-9' & - & - & - & - & - & - & - & - & 24,72 & 24,90 \\
\hline H-7 & 7,80 & 7,50 & 7,76 & 7,60 & 7,59 & 7,50 & 7,84 & 7,41 & 7,82 & 7,33 \\
\hline H-8 & 7,48 & 7,01 & 7,83 & 7,30 & - & - & - & - & - & - \\
\hline $\mathrm{H} 2^{\prime} / \mathrm{H} 6^{\prime}$ & 8,02 & - & 8,01 & - & 7,83 & 7,90 & 8,10 & 8,14 & 7,76 & 7,73 \\
\hline H-7' & - & - & - & - & 3,89 & 3,80 & 3,10 & 2,79 & 2,88 & 2,84 \\
\hline H- 8 & - & - & - & - & - & - & 2,90 & 2,98 & 2,06 & 2,34 \\
\hline H-9, & - & - & - & - & - & - & - & - & 2,58 & 1,88 \\
\hline
\end{tabular}

${ }^{\mathrm{a} D e s l o c a m e n t o s}$ químicos de carbonos $\left(\delta_{\mathrm{C}}\right)$ não assinalados devido à multiplicidade produzida pelo acoplamento com átomos de flúor. ${ }^{\mathrm{b}}$ Deslocamentos químicos de carbonos $\left(\delta_{\mathrm{C}}\right)$ quaternários não assinalados devido à pequena quantidade de amostra e ao número reduzido de acumulações.

Tabela 3. Variação $(\Delta \delta)$ dos deslocamentos químicos de H-2', H-8, H-7,C-2', C-8, C-7 e C-9 para as substâncias 2,3 e 4 em relação à chalcona (1) e $\mathbf{6 , 8}$ e $\mathbf{1 0}$ relacionadas com $\mathbf{5}, 7$ e $\mathbf{9}$, respectivamente.

\begin{tabular}{cccccccc}
\hline & $\mathbf{2}-\mathbf{1}$ & $\mathbf{3}-\mathbf{1}$ & $\mathbf{4}-\mathbf{1}$ & $\mathbf{6}-\mathbf{5}$ & $\mathbf{8}-\mathbf{7}$ & $\mathbf{1 0}-\mathbf{9}$ \\
\hline H-8 & $-0,47$ & 0,35 & $-0,18$ & - & - & - \\
H-7 & $-0,30$ & $-0,04$ & $-0,18$ & $-0,09$ & $-0,43$ & $-0,49$ \\
H-2 & $\mathrm{a}$ & $-0,01$ & $\mathrm{a}$ & 0,07 & 0,04 & $-0,03$ \\
C-8 & 4,06 & 6,80 & 9,96 & $\mathrm{a}$ & $\mathrm{A}$ & $\mathrm{a}$ \\
C-7 & 3,50 & $-17,05$ & $-14,62$ & $-16,65$ & $-16,56$ & $-16,73$ \\
C-2 & $\mathrm{b}$ & 0,20 & $\mathrm{~b}$ & 0,07 & $-0,04$ & 0,07 \\
C-9 & $-6,69$ & $-1,18$ & $-7,54$ & $-1,55$ & $-1,07$ & $-1,85$ \\
\hline
\end{tabular}

${ }^{a}$ Estes sinais não foram assinalados devido à multiplicidade produzida pela interação destes carbonos com os átomos de flúor $\left({ }^{\mathrm{n}} \mathrm{J}_{\mathrm{CF}}, \mathrm{n}=1,2,3\right.$ e 4$) .{ }^{\mathrm{b}} \mathrm{O}$ valor de $\Delta \delta$ não foi calculado pela falta do deslocamento químico do derivado fluorado (a).

Tabela 4. Dados de deslocamento químico de $\mathrm{RMN}{ }^{1} \mathrm{H}(\delta)$ para os compostos $\mathbf{1 - 4}$ em $\mathrm{CDCl}_{3}$ com TMS como referência interna e $\mathrm{CCl}_{4}$ com $\mathrm{CDCl}_{3}$ e TMS para ajuste do Lock e referência externa, respectivamente, e variação dos deslocamentos químicos $(\Delta \delta)$ de $\mathrm{H}_{7}$ e $\mathrm{H}_{8}$ das substâncias $\mathbf{2 - 4}$ em relação à chalcona (1) em $\mathrm{CDCl}_{3}$ ou $\mathrm{CCl}_{4}$ como solvente.

\begin{tabular}{cccccccccc}
\hline & \multicolumn{3}{c}{$\mathrm{CHCl}_{3}$} & & \multicolumn{3}{c}{$\mathrm{CCl}_{4}$} \\
\hline$\delta$ & $\mathbf{1}$ & $\mathbf{2}$ & $\mathbf{3}$ & $\mathbf{4}$ & $\mathbf{1}$ & $\mathbf{2}$ & $\mathbf{3}$ & $\mathbf{4}$ \\
\hline $\mathrm{H}_{7}$ & 7,80 & 7,50 & & 7,76 & 7,62 & 7,86 & 7,69 & 7,88 & 7,69 \\
$\mathrm{H}_{8}$ & 7,48 & 7,01 & & 7,83 & 7,30 & 7,56 & 7,13 & 7,98 & 7,45 \\
\hline$\Delta \delta$ & $\mathbf{2 - 1}$ & & $\mathbf{3 - 1}$ & $-0,04$ & $\mathbf{4 - 1}$ & $\mathbf{2 - 1}$ & $\mathbf{3 - 1}$ & $\mathbf{4 - 1}$ & $-0,17$ \\
\hline $\mathrm{H}_{7}$ & $-0,30$ & $-0,35$ & $-0,18$ & $-0,17$ & 0,02 & $-0,11$ \\
$\mathrm{H}_{8}$ & $-0,47$ & & 0,18 & $-0,43$ & & 0,42 & \\
\hline
\end{tabular}

tuintes. Para os carbonos, os espectros de $\mathrm{RMN}{ }^{13} \mathrm{C}$ (HBBD totalmente desacoplado e DEPT) de 1 mostraram que o C-7 absorve em campo mais baixo do que o $\mathrm{C}-8$, como previsto pelo efeito de desproteção por mesomeria exercido pela carbonila. Os efeitos gerados pela presença de substituintes polares no anel aromático (anel A) pertencente ao grupo cinamoíla são transmitidos por efeito de ressonância para H-8 e C-8 e por mecanismo indutivo para $\mathrm{H}-7$ e C-7. Por outro lado, substituintes sustentados pelo anel aromático (anel B) ligado ao carbono carbonílico exercem efeito indutivo maior ${ }^{24}$ em $\mathrm{H}-8$ e C-8 do que em H-7 e C-7.

No espectro bidimensional de correlação heteronuclear de hidrogênio e carbono através de uma ligação $\left({ }^{13} \mathrm{Cx}^{1} \mathrm{H}-\mathrm{COSY}\right.$ ${ }^{1} \mathrm{~J}_{\mathrm{CH}}$ ) da chalcona 1 constatou-se, por leitura direta de pico transversal, que o sinal de maior deslocamento químico $\left(\delta_{\mathrm{H}} 7,80\right)$ representa o hidrogênio que está ligado ao carbono 
Tabela 5. Dados obtidos dos espectros infravermelho (IV) das substâncias 1-10, em $\mathrm{CCl}_{4}$ e 1-4, em $\mathrm{CHCl}_{3}$.

\begin{tabular}{lcccccccccc}
\hline $\begin{array}{l}\text { número de onda } \\
\left(\mathrm{cm}^{-1}\right) / \mathrm{CCl}_{4}\end{array}$ & $\mathbf{1}$ & $\mathbf{2}$ & $\mathbf{3}$ & $\mathbf{4}$ & $\mathbf{5}$ & $\mathbf{6}$ & $\mathbf{7}$ & $\mathbf{8}$ & $\mathbf{9}$ & $\mathbf{1 0}$ \\
\hline $\mathrm{C}=\mathrm{O}$ s-cis & 1670 & 1682 & 1677 & 1690 & 1670 & 1680 & 1670 & 1678 & 1662 & 1680 \\
$\mathrm{C}=\mathrm{O}$ s-trans & 1647 & 1665 & 1650 & 1675 & - & - & - & - & \\
$\mathrm{C}=\mathrm{C}$ s-cis & $\mathrm{a}$ & 1625 & $\mathrm{a}$ & 1625 & $\mathrm{a}$ & 1650 & $\mathrm{a}$ & 1650 & $\mathrm{a}$ & 1650 \\
$\mathrm{C}=\mathrm{C}$ s-trans & $\mathrm{a}$ & 1650 & $\mathrm{a}$ & 1647 & - & - & - & - & - \\
\hline número de onda & $\mathbf{1}$ & $\mathbf{2}$ & $\mathbf{3}$ & $\mathbf{4}$ & $\mathbf{5}$ & $\mathbf{6}$ & $\mathbf{7}$ & $\mathbf{8}$ & $\mathbf{9}$ & $\mathbf{1 0}$ \\
$\left(\mathrm{cm}^{-1}\right) / \mathrm{CHCl}_{3}$ & & & & & & & & - & - & - \\
\hline $\mathrm{C}=\mathrm{O}$ s-cis & 1664 & 1677 & 1672 & 1689 & - & - & - & - & - \\
$\mathrm{C}=\mathrm{O}$ s-trans & 1639 & 1663 & 1648 & 1673 & - & - & - & - & - \\
\hline
\end{tabular}

${ }^{a}$ Banda sobreposta à banda de respiração do anel aromático na região de $1600 \mathrm{~cm}^{-1}$.

olefínico de maior deslocamento químico C-7 $\left(\delta_{\mathrm{C}} 144,71\right)$, definindo-se assim o sinal correspondente ao H-7. Já o sinal de menor deslocamento químico $\left(\delta_{\mathrm{H}} 7,48\right)$, observado na região de absorção dos hidrogênios aromáticos, está correlacionado com o carbono C-8 que apresenta deslocamento químico em $\left(\delta_{\mathrm{C}} 121,99\right)$ e, conseqüentemente, representa o H-8. Estes resultados estão em acordo com os valores descritos na literatura ${ }^{36,37}$.

Com base nos mesmos efeitos, a análise dos espectros de RMN das substâncias 5 e $6(n=1), 7$ e $8(n=2)$ e 9 e $10(n=3)$, análogos cíclicos de chalcona de configuração $E$ e nos quais somente a conformação $s$-cis é possível, contribuiu para uma melhor compreensão dos estudos em questão. A existência de uma única conformação simplificou em muito as nossas deduções. A configuração $E$ destes produtos (5-10) foi confirmada através de espectro ${ }^{1} \mathrm{Hx}{ }^{1} \mathrm{H}-\mathrm{NOE}$ de 7 e de experiências de efeito nuclear Overhauser $(\mathrm{NOE}=$ Nuclear Overhauser Effect) por subtração de espectros. ${ }^{38}$ Esta substância foi escolhida por apresentar estrutura intermediária entre os derivados cíclicos (5-10).

A análise dos espectros de $\mathrm{RMN}{ }^{13} \mathrm{C}$ de 5, 7 e 9 (Tabela 2) permitiu constatar o aparecimento dos sinais referentes aos carbonos carbonílicos de $\mathbf{5}\left(\delta_{\mathrm{C}} 194,04, \Delta \delta_{\mathrm{C}}=+6,79\right)$ e $9\left(\delta_{\mathrm{C}}\right.$ $\left.197,98, \Delta \delta_{\mathrm{C}}=+10,73\right)$ com maiores deslocamentos químicos quando comparados com o valor obtido para a substância $7\left(\delta_{C}\right.$ 187,24). Considerando-se somente o tamanho do anel, estes dados não obedecem à mesma ordem verificada na comparação dos deslocamentos químicos dos carbonos carbonílicos da ciclopentanona $11\left(\delta_{\mathrm{C}} 219,6\right)>$ cicloeptanona $13\left(\delta_{\mathrm{C}} 215,0\right)>$ cicloexanona $12\left(\delta_{\mathrm{C}} 209,7\right)^{38}$.

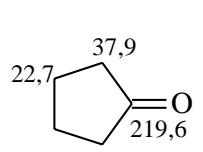

11

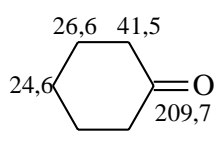

12

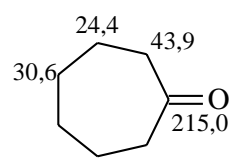

13
A variação observada nos deslocamentos químicos dos carbonos carbonílicos (C-9) de 5, 7 e 9 pode ser justificada pelas alterações na densidade eletrônica destes átomos, provocadas provavelmente por mudanças nas interferências estéricas e/ou tensão dos ângulos de ligação que respondem pela conformação mais estável. Um aumento na coplanaridade da carbonila, tanto com o grupo benzilideno quanto com o anel aromático $\mathrm{B}$, leva a um aumento na conjugação com o sistema de elétrons $\pi$, aumentando com isso a densidade eletrônica sobre o carbono carbonílico. Desta forma, pode-se admitir que para a substância $9(\mathrm{n}=3)$ há uma menor coplanaridade e, conseqüentemente, menor deslocalização eletrônica e maior deslocamento químico $\left(\delta_{C} 197,98\right)$ na sua conformação mais estável, quando comparado com a substância $\mathbf{7}$, que revela o sinal do carbono carbonílico com menor deslocamento químico $\left(\delta_{\mathrm{C}} 187,24\right)$. Apesar de 5 possuir a estrutura mais plana entre estas três cetonas $\alpha, \beta$-insaturadas (Tabela 6), o seu carbono carbonílico aparece com maior deslocamento químico $\left(\delta_{C} 194,04\right)$ do que o de $7\left(\delta_{\mathrm{C}} 187,24\right)$ devido à tensão do anel de cinco membros. Isto pode ser facilmente observado na comparação entre os deslocamentos químicos dos carbonos carbonílicos da 2ciclopentenona $14\left(\delta_{\mathrm{C}} 209,8\right)$ e 2-cicloexenona $\mathbf{1 5}\left(\delta_{\mathrm{C}} 199,0\right){ }^{38}$<smiles>O=C1C=CCC1</smiles>

14<smiles>O=C1CCCCCC1</smiles>

15
A modificação observada nos deslocamentos químicos de C-7 e C-2' com o aumento de n (Tabela 2), provavelmente reflete, além do efeito mesomérico, uma diminuição do efeito de proteção $\gamma$ exercido pelo oxigênio carbonílico nestes dois carbonos, em decorrência dos ângulos diedros $\mathrm{O}=\mathrm{C}-\mathrm{C} 7=\mathrm{C} 8 \mathrm{e}$ $\mathrm{O}=\mathrm{C}-\mathrm{C} 1{ }^{\prime}=\mathrm{C} 2$ ', que podem ser alterados pelas modificações conformacionais em função do aumento de n (Tabela 6).

Tabela 6. Valores dos ângulos de ligação $(\theta)$ e ângulos diedro $\left(\theta^{\prime}\right)$ observados nas substâncias 1-10. ${ }^{\text {a }}$

\begin{tabular}{lcccccccccc}
\hline Substâncias & $\mathbf{1}$ & $\mathbf{2}$ & $\mathbf{3}$ & $\mathbf{4}$ & $\mathbf{5}$ & $\mathbf{6}$ & $\mathbf{7}$ & $\mathbf{8}$ & $\mathbf{9}$ & $\mathbf{1 0}$ \\
\hline Ligações & $\theta$ & $\theta$ & $\theta$ & $\boldsymbol{\theta}$ & $\boldsymbol{\theta}$ & $\boldsymbol{\theta}$ & $\boldsymbol{\theta}$ & $\boldsymbol{\theta}$ & $\boldsymbol{\theta}$ & $\theta$ \\
\hline C1'-C9-C8 & 116,37 & 114,94 & 116,35 & 115,14 & 108,11 & 107,17 & 118,76 & 119,04 & 120,53 & 120,46 \\
C1'-C9-O & 121,18 & 120,81 & 121,55 & 121,01 & 125,44 & 125,99 & 120,66 & 120,45 & 118,62 & 118,67 \\
C9-C8-C7 & 120,81 & 121,06 & 120,62 & 120,84 & 124,17 & 124,84 & 121,81 & 121,63 & 121,05 & 121,06 \\
C9-C1'-C2' & 121,92 & 120,53 & 121,88 & 120,51 & 129,59 & 129,03 & 121,12 & 121,10 & 119,44 & 119,53 \\
O-C9-C8 & 122,43 & 124,25 & 122,09 & 123,86 & 126,45 & 126,83 & 120,58 & 120,51 & 120,85 & 120,87 \\
\hline Diedros & $\theta^{\prime}$ & $\theta^{\prime}$ & $\theta^{\prime}$ & $\theta^{\prime}$ & $\theta^{\prime}$ & $\theta^{\prime}$ & $\theta^{\prime}$ & $\theta^{\prime}$ & $\theta^{\prime}$ & $\theta^{\prime}$ \\
\hline C2'-C1'-C9-O & 32,82 & 57,91 & 32,58 & 58,07 & 0,22 & 0,55 & 7,83 & 9,72 & 39,53 & 40,95 \\
O-C9-C8-C7 & 40,72 & 39,71 & 43,79 & 43,64 & 1,96 & 1,62 & 19,96 & 16,78 & 18,01 & 15,96 \\
C8-C7-C1-C2 & $-35,55$ & $-35,42$ & $-43,82$ & $-43,59$ & $-59,10$ & $-72,68$ & $-70,04$ & $-68,05$ & $-82,02$ & $-90,67$ \\
\hline
\end{tabular}

${ }^{\text {a }}$ calculados pela utilização do programa PC-MODEL 
Uma comparação entre os valores dos deslocamentos químicos dos carbonos encontrados para os derivados 5-10 com aqueles da chalcona (1) e de seus derivados fluorados 2-4 não foi considerada porque a presença do grupo $\mathrm{CH}_{2}$ em 5-10 influencia os deslocamentos químicos tanto de C-8 (efeito $\alpha$ ) quanto de C-7 (efeito $\beta$ ), como pode ser deduzido pelas diferenças estruturais apresentadas para estas duas classes de substâncias (Esquema 1).

Em relação aos valores encontrados para os deslocamentos químicos de $\mathrm{H}-7$ e $\mathrm{H}-2$ ', pode-se interpretá-los como descrito a seguir. $\mathrm{O}$ H-2' encontra-se ligado diretamente a um anel aromático B (6 elétrons $\pi$ em um sistema cíclico) enquanto que o C-7 está ligado a uma ligação dupla (2 elétrons $\pi$ ), justificando a maior desproteção anisotrópica sentida pelo $\mathrm{H}-2$ ' (maior deslocamento químico).

Obviamente, os deslocamentos químicos para estes hidrogênios H-2' e H-7 dependem também da contribuição anisotrópica exercida pelo grupo carbonila e, conseqüentemente, das suas posições ocupadas em relação ao cone de anisotropia correspondente.

Através de cálculos teóricos utilizando o programa PCMODEL, observou-se que os ângulos de ligação $(\theta)$, bem como a comparação entre os ângulos diedros formados pelas ligações $\mathrm{C} 1^{\prime}=\mathrm{C} 2^{\prime}-\mathrm{C}=\mathrm{O}\left(\theta-1^{\prime}\right)$ com $\mathrm{C} 7=\mathrm{C} 8-\mathrm{C}=\mathrm{O}\left(\theta-2^{\prime}\right)$ (Tabela 6$)$, podem ser utilizados para justificar as variações dos deslocamentos químicos de H-2' e H-7 pela influência anisotrópica do grupo carbonila.
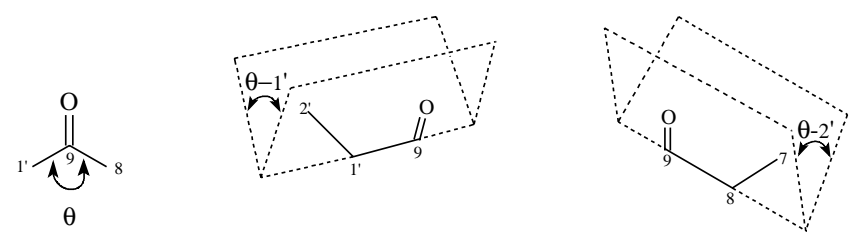

Assim, considerando-se a substância 7 como referência [ângulo de ligação C1'-C9-C8 $=118,76^{\circ}, \delta_{\mathrm{H}} 8,10\left(\mathrm{H}-2^{\prime}\right)$ e 7,84 (H-7)], pode-se justificar os menores deslocamentos químicos de H-2' $\left(\delta_{\mathrm{H}} 7,83\right)$ e H-7 $\left(\delta_{\mathrm{H}} 7,59\right)$ na substância 5 , devido ao menor ângulo de ligação $\mathrm{C} 1^{\prime}-\mathrm{C} 9-\mathrm{C} 8=108,11^{\circ}$ (maiores distâncias de H-2' e H-7 do efeito anisotrópico de $\mathrm{C}=\mathrm{O}$ ) e ângulos diedros $\mathrm{C} 1^{\prime}-\mathrm{C} 2^{\prime}-\mathrm{C}=\mathrm{O}\left(0,22^{\circ}\right)$ e $\mathrm{C} 8-\mathrm{C} 7-\mathrm{C}=\mathrm{O}\left(1,96^{\circ}\right)$, com H-2' e H-7 próximos do plano da função carbonila. No caso da substância 9, os ângulos respectivos (de ligação 120,53: menores distâncias de H-2' e H-7 do efeito anisotrópico da carbonila; diedros $\theta-1^{\prime} 39,52^{\circ}$ e $\theta-2^{\prime} 18,01^{\circ}$ : maiores distâncias do plano do grupo carbonila) demonstram que tais hidrogênios afastam-se da influência anisotrópica do grupo carbonila, sendo que H-2' $\left(\theta-1^{\prime}=39,52^{\circ}\right)$ alcança maior distância e tem menor deslocamento químico do que H-7, como conseqüência da ocupação de região de menor efeito desprotetor (região de proteção).

A comparação das substâncias fluoradas $\mathbf{6 , 8}$ e 10 entre si, e com 5, 7 e 9 (Tabela 3), permitiu verificar resultados análogos e, conseqüentemente, a ocorrência de efeitos semelhantes. Assim, a interação estérica do átomo de flúor com o grupo metilênico alílico do sistema heptacíclico em 10 introduz uma modificação conformacional que conduz o H-7 para a região de proteção anisotrópica do grupo carbonila, justificando-se o menor deslocamento químico deste próton $\left(\theta_{\mathrm{H}} 7,33\right)$ quando comparado com o de $\mathrm{H}-7$ de $9\left(\theta_{\mathrm{H}} 7,82\right)$.

Efetuando-se a comparação entre os dados espectrais para $\mathbf{5 / 6}, \mathbf{7 / 8}$ e 9/10 (Tabela 3) é possível obter informações relativas à influência dos átomos de flúor no deslocamento químico dos principais átomos de hidrogênio e carbono classificados como os mais informativos sob o ponto de vista estrutural, isto é, H-2', H-7, C-2', C-7 e C=O. Como os átomos de flúor são doadores de elétrons por mesomeria e retiradores por efeito indutivo, os valores calculados para a variação dos deslocamentos químicos $(\Delta \delta)$ com base nestes pares de substâncias permitem avaliar a influência destes efeitos eletrônicos.

A análise direta das diferenças de deslocamento químico $(\Delta \delta)$ permitiu deduzir que o efeito mesomérico doador de elétrons do flúor assume predominância em relação ao efeito indutivo retirador de elétrons. Esta dedução apoiou-se experimentalmente nos menores valores observados para os deslocamentos químicos do átomos de carbono carbonílico C-9 e C-7 e para o átomo de hidrogênio $\mathrm{H}-7$ nos derivados fluorados $\mathbf{6}$, 8 e 10, quando comparados com os dos compostos 5, 7 e $\mathbf{9}$, respectivamente (Tabela 2).

Diante destas informações, foi possível então interpretar com segurança os dados espectrais de RMN para os derivados fluorados 2-4 (Tabela 2). Considerando-se os valores de deslocamento químico encontrados para os carbonos C-9 (carbonílico), C-2'/C-6', C-8 e C-7 e os hidrogênios H-2'/H-6', H-7 e H-8 pode-se entender as modificações observadas na comparação dos dados para os derivados fluorados com os da chalcona 1 (Tabela 3).

Considerando-se a fraca influência decorrente da substituição de $\mathrm{H}-8$ por um grupo $\mathrm{CH}_{2}$ sobre o deslocamento químico dos núcleos de hidrogênio pode-se fazer uma comparação entre os valores de deslocamento químico encontrados para os hidrogênios H-2' e H-7 de 7 e da chalcona (1). Assim, para os hidrogênios H-7 (1 e 7), H-2' (7) e H-2'/H-6' (1) destas duas substâncias (Tabela 1) observou-se deslocamentos químicos praticamente iguais, podendo-se considerar esses dados como um indicativo de geometrias muito semelhantes para as duas moléculas e, conseqüentemente, postular a conformação s-cis como adotada predominantemente por chalcona (1). Esses resultados juntamente com aqueles obtidos para a constante de acoplamento $(\sim 16 \mathrm{~Hz})$ correspondente à interação spin-spin de H-7 com H-8, permitiram definir a configuração $E$ (termodinamicamente mais estável) para chalcona (1) e seus derivados 2-4.

No espectro bidimensional de correlação heteronuclear de hidrogênio e carbono-13 através de uma ligação $\left({ }^{13} \mathrm{CX}^{1} \mathrm{H}\right.$ COSY ${ }^{1}{ }^{1} \mathrm{~J}_{\mathrm{CH}}$ ) de 2 constatou-se a presença de pico transversal revelando que o sinal de hidrogênio de maior deslocamento químico $\left(\delta_{\mathrm{H}} 7,50\right)$ correlaciona-se com o sinal do carbono C-7 $\left(\delta_{\mathrm{C}} 148,21\right)$, com o sinal em $\delta_{\mathrm{H}} 7,50$ sendo então atribuído a H-7. Já o sinal de hidrogênio de menor deslocamento químico $\left(\delta_{\mathrm{H}} 7,01\right)$, observado na região dos hidrogênios aromáticos, aparece correlacionado com o do C-8 $\left(\delta_{\mathrm{C}} 126,06\right)$, podendo ser definido como representante do hidrogênio H-8. Comparandose estes resultados com aqueles obtidos para chalcona (1) (Tabela 3), pode-se notar uma variação nos deslocamentos quími$\cos$ dos carbonos $\mathrm{C}=\mathrm{O}, \mathrm{C}-8$ e $\mathrm{C}-7$, sendo mais pronunciada a proteção no carbono $\mathrm{C}=\mathrm{O}\left(\Delta \delta_{\mathrm{C}}=-6,69\right)$, em direção oposta à desproteção dos carbonos CH-8 $\left(\Delta \delta_{\mathrm{C}}=+4,06\right)$ e CH-7 $\left(\Delta \delta_{\mathrm{C}}=\right.$ $+3,50)$. A proteção $\left(\Delta \delta_{C}=-6,69\right)$ revelada pela modificação do deslocamento químico do carbono carbonílico [ $\delta_{C} 190,40$ (1) e 183,70 (2)] pode ser justificada pelo efeito mesomérico exercido pelos átomos de flúor, como demonstram as estruturas canônicas descritas no Esquema 2, utilizando-se somente um dos átomos de flúor (os átomos de flúor orto e para contribuem para o híbrido de ressonância).

$\mathrm{O}$ efeito dos átomos de flúor de doar elétrons por mesomeria atenua o efeito retirador de elétrons por ressonância do grupo carbonila sobre a ligação dupla, ocorrendo então uma diminuição na densidade de elétrons no carbono $\mathrm{C}-8$, refletida pelo maior deslocamento químico observado [ $\delta_{\mathrm{C}} 121,99$ (1) para 126,06 (2)]. Além disto, deve-se considerar também a interferência estérica entre os átomos de flúor localizados nos carbonos C-2'/C-6' e o átomo de hidrogênio $\mathrm{H}-8$, assim como a repulsão eletrônica decorrente do aumento de densidade eletrônica no átomo de oxigênio carbonílico com os elétrons $\pi$ da ligação dupla na conformação $s$-cis, favorecendo o estereoisômero s-trans. 


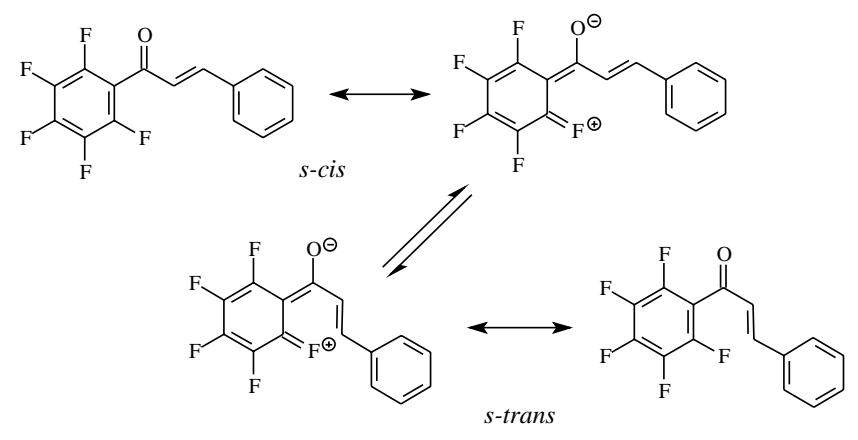

Esquema 2.

Nesta conformação $s$-trans o efeito $\gamma$ protetor exercido pelo carbono C-2'/C-6' sobre C-8 perde eficiência devido à orientação oposta do H-8, contribuindo também para o maior deslocamento químico do C-8. Além disto, na conformação $s$-trans desaparece a condição favorável observada na conformação $s$ cis para o efeito $\gamma$ protetor do oxigênio do grupo carbonila sobre o carbono C-7, justificando o maior deslocamento químico deste carbono em $2\left(\delta_{\mathrm{C}} 148,26\right)$ quando comparado com o da chalcona $\left(\mathbf{1}, \delta_{\mathrm{C}} 144,78\right)$.

Um forte efeito de proteção é observado tanto para $\mathrm{H}-8$ como para H-7 $\left(\Delta \delta_{\mathrm{H}}=0,47\right.$ e $\Delta \delta_{\mathrm{H}}=0,30$, respectivamente $)$ em 2. Estas modificações $\left(\Delta \delta_{\mathrm{H}}\right)$ nos deslocamentos químicos para estes átomos de hidrogênio de $\mathbf{2}$ também refletem uma mudança nas porcentagens $s$-cis/s-trans do equilíbrio conformacional. Estes dados estão em acordo com os argumentos utilizados acima e podem justificar a redução do efeito retirador de elétrons do grupo carbonila por mesomeria, sendo que no caso dos hidrogênios H-8 e H-7 deve-se considerar também a redução do efeito anisotrópico exercido pela carbonila. Pode-se verificar também que H-8 na conformação $s$-cis sofre maior efeito de desproteção anisotrópica por se encontrar em região de desproteção promovida pelos dois anéis aromáticos. Isto não ocorre na conformação $s$-trans, uma vez que nesta geometria este hidrogênio se situa entre uma carbonila e um anel aromático. Este fato contribui para uma diminuição da desproteção por anisotropia sofrida por H-8. O H-7 na conformação $s$-cis está na região de desproteção da carbonila e do anel aromático A. Na conformação s-trans, a carbonila deixa de exercer a desproteção significativa sobre este H-7, assumindo posição cis em relação ao núcleo aromático $\mathrm{B}$ que, por razões estéricas, pode ser afastado do plano da molécula para assumir uma orientação onde exerça proteção sobre H-7, contribuindo para o menor deslocamento químico $\left(\delta_{\mathrm{H}} 7,50\right)$ observado para este hidrogênio quando comparado com o da chalcona (1) $\left(\delta_{\mathrm{H}} 7,80\right)$.

A Tabela 3 revela modificação para menor valor de $\delta_{C}$, aproximadamente $\Delta \delta_{C}=-16,5$, quando o deslocamento químico de C-7 nos análogos cíclicos das chalconas não fluoradas $(5,7$ e 9) é comparado com o deslocamento químico de C-7 nos compostos benzilideno-pentafluorados $\mathbf{6 , 8}$ e $\mathbf{1 0}$. Com base nesses resultados, tornou-se possível atribuir a $\mathrm{C}-7$ o sinal em $\delta_{\mathrm{C}} 127,66$ no espectro de RMN ${ }^{13} \mathrm{C}$ de 3 . O espectro de ${ }^{13} \mathrm{CX}{ }^{1} \mathrm{H}$ $-\operatorname{COSY}{ }^{-1} J_{\mathrm{CH}}$ para esta substância permitiu definir os deslocamentos químicos dos hidrogênios $\mathrm{H}-7\left(\delta_{\mathrm{H}} 7,76\right)$, correlacionado com o sinal em $\delta_{\mathrm{C}} 127,66$, e H-8 $\left(\delta_{\mathrm{H}} 7,83\right)$ com o do carbono $\mathrm{C}-8\left(\delta_{\mathrm{C}} 128,79\right)$.

As modificações observadas nos deslocamentos químicos de H-8 e H-7 do composto 3 podem ser interpretadas como um aumento da concentração da conformação $s$-cis no equilíbrio conformacional, já que uma maior porcentagem desta conformação deve aumentar o efeito de desproteção sentido por H-8 (efeito anisotrópico dos dois anéis aromáticos). Por outro lado, o efeito mesomérico doador de elétrons dos átomos de flúor justifica os menores deslocamentos químicos para $\mathrm{H}-7$ e C-7 quando comparados com os valores observados para chalcona (1). O efeito $\gamma$ protetor do oxigênio do grupo carbonila na conformação $s$-cis contribui também para o sinal de C-7 aparecer com menor deslocamento químico (Tabela 2).

Finalmente, utilizando-se os dados espectrais interpretados até aqui, foi possível estabelecer a atribuição dos deslocamentos químicos de $\mathrm{H}-8, \mathrm{H}-7, \mathrm{C}-8, \mathrm{C}-7$ e $\mathrm{C}=\mathrm{O}$ para o derivado 4. $\mathrm{O}$ espectro ${ }^{13} \mathrm{Cx}^{1} \mathrm{H}-\mathrm{COSY}{ }^{1} \mathrm{~J}_{\mathrm{CH}}$ de $\mathbf{4}$ permitiu definir a correlação de H-8 $\left(\delta_{\mathrm{H}} 7,30\right)$ com C-8 $\left(\delta_{\mathrm{C}} 131,95\right)$ e de H-7 $\left(\delta_{\mathrm{H}} 7,62\right)$ com C-7 $\left(\delta_{C} 130,09\right)$. A confirmação desta dedução foi obtida pela constante de acoplamento $\left(\mathrm{J}_{\mathrm{CF}}=8,5 \mathrm{~Hz}\right)$, observada no sinal tripleto correspondente ao $\mathrm{C}-8\left(\delta_{\mathrm{C}} 131,95\right)$. Este valor para a constante de acoplamento entre os átomos de carbono e flúor indica uma interação spin-spin através do espaço. Também foram observados acoplamentos entre $\mathrm{H}-8$ e os flúor presentes em C-2' e C-6' para os compostos 2 e 4, com valores de $\mathrm{J}_{\mathrm{HF}}=1,1$ e $1,7 \mathrm{~Hz}$, respectivamente, os quais também ocorrem por uma interação através do espaço ${ }^{38,39}$.

As modificações observadas nos deslocamentos químicos de H-8, H-7, C-8 e C-7 de 4, quando comparados com os valores encontrados para chalcona (1), podem ser justificadas pelos resultados obtidos para os derivados 2 e $\mathbf{3}$. Os valores obtidos para os deslocamentos químicos dos átomos de carbono C-8, C-7 e C-9 obedecem às previsões deduzidas dos valores de $\Delta \delta$ encontrados para 2 e 3. O deslocamento químico do átomo de carbono C-8 nestas chalconas fluoradas revela desproteção em relação ao da chalcona (1) (Tabela 3), no composto 2 por $\Delta \delta_{\mathrm{C}}=+4,06$ e, no composto 3 , por $\Delta \delta_{\mathrm{C}}=+6,80$ o mesmo ocorrendo com o C- 8 $\left(\Delta \delta_{\mathrm{C}}=+9,96\right)$ de 4 . Somando-se estes dois valores obtidos para 2 e 3 ao deslocamento químico de C-8 $\left(\delta_{C} 121,99\right)$ para chalcona (1) obtém-se $\delta_{\mathrm{C}} 132,85$, valor próximo ao obtido experimentalmente para o derivado $4\left(\delta_{\mathrm{C}} 131,95\right)$. Para o carbono C-7, os valores de $\Delta \delta_{\mathrm{C}}$ encontrados para $2\left(\Delta \delta_{\mathrm{C}}=+3,50\right)$ e $\mathbf{3}\left(\Delta \delta_{\mathrm{C}}=\right.$ $-17,05)$ indicam desproteção e proteção, respectivamente. Efetuando-se a soma algébrica desses valores com o encontrado para o deslocamento químico de C-7 $\left(\delta_{\mathrm{C}} 144,71\right)$ de chalcona $(\mathbf{1})$, obtém-se $\delta_{\mathrm{C}} 131,16$, valor que está de acordo com o valor experimental de $\delta_{\mathrm{C}} 130,09$. Procedimento idêntico, isto é, usando a soma algébrica dos $\Delta \delta_{\mathrm{C}}$ observados nos deslocamentos químicos dos carbonos carbonílicos de $\mathbf{2}\left(\Delta \delta_{\mathrm{C}}=-6,69\right)$ e $\mathbf{3}\left(\Delta \delta_{\mathrm{C}}=-1,18\right)$ com o da chalcona $(1)\left(\delta_{\mathrm{C}} 190,40\right)$, obteve-se $\delta_{\mathrm{C}} 182,52$, também de acordo com o valor experimental observado para $4\left(\delta_{\mathrm{C}} 182,86\right)$.

Para explicar as modificações verificadas nos deslocamentos químicos dos hidrogênios C-8 e C-7 de $\mathbf{4}$, em relação à chalcona (1), foi necessária uma interpretação envolvendo os efeitos causados pela substituição de $\mathrm{H}$ por $\mathrm{F}$ em ambos os anéis. Como deduzido anteriormente, a presença de átomos de flúor nos carbonos orto do anel aromático B (ligado ao carbono carbonílico) afasta esta unidade do plano da molécula para atenuar a interferência estérica entre os átomos de flúor e H-8. Esta situação espacial contribui para o aumento da concentração da conformação $s$-trans no equilíbrio conformacional, além de diminuir a capacidade retiradora de elétrons do grupo carbonila (devido à ressonância com os átomos de flúor em orto e para) por mesomeria sobre a ligação dupla. Assim, H8 apresenta um deslocamento químico intermediário quando comparado com os compostos $\mathbf{2}$ e $\mathbf{3}$, como decorrência da substituição por flúor em ambos os anéis aromáticos. A susbtituição no anel B provoca um efeito de proteção de $0,47 \mathrm{ppm}$, enquanto que a substituição no anel A leva à desproteção por $\Delta \delta=-0,15$. Aplicando-se esta diferença para o caso do composto 4 , obtém-se um $\delta_{\text {teórico }}=7,16$. Por outro lado, H-7 em 4 $\left(\delta_{\mathrm{H}} 7,60\right)$ torna-se mais protegido do que $\mathrm{H}-7$ da chalcona $(\mathbf{1})$ $\left(\delta_{\mathrm{H}} 7,80\right)$ devido ao aumento da densidade eletrônica no carbono C-7 provocado pelo efeito mesomérico exercido pelos átomos de flúor do anel $\mathrm{A}$.

No sentido de confrontar os resultados obtidos nos estudos de RMN com os de espectroscopia na região do infravermelho, foram realizados estudos de RMN ${ }^{1} \mathrm{H}$ em solução de $\mathrm{CCl}_{4}$ 
e TMS em $\mathrm{CDCl}_{3}$ como referência externa. Os valores encontrados para os deslocamentos químicos dos derivados 1-4 realizados em $\mathrm{CCl}_{4}$ estão de acordo com aqueles obtidos em $\mathrm{CHCl}_{3}$ (Tabela 4). Suas interpretações seguem o mesmo raciocínio usado na análise dos espectros obtidos em $\mathrm{CHCl}_{3}$ como solvente e, conseqüentemente, só podemos justificar os deslocamentos químicos observados para $\mathrm{RMN}{ }^{1} \mathrm{H}$ se levarmos em conta as mudanças nas porcentagens das concentrações $s$-cis/strans dos confôrmeros em equilíbrio quando comparados à chalcona 1. Os maiores deslocamentos químicos encontrados em $\mathrm{CCl}_{4}$ (núcleos menos blindados) quando comparados aos valores obtidos em $\mathrm{CHCl}_{3}$ indicam que em solventes apolares os compostos carbonílicos $\alpha, \beta$-insaturados devem apresentar estruturas com um maior grau de coplanaridade. Já as variações de deslocamentos $\Delta \delta$ em relação àquelas apresentadas por chalcona (1) em $\mathrm{CHCl}_{3}$, quando comparado aos resultados em $\mathrm{CCl}_{4}$, indicam uma maior porcentagem do confôrmero menos polar no equilíbrio conformacional em $\mathrm{CCl}_{4}$, o que pode ser racionalizado assumindo-se um menor efeito de proteção no caso do derivado $2\left(\Delta \delta \alpha=-0,47\right.$ e $\Delta \delta \beta=-0,30 \mathrm{em}^{-C_{C l}}$ e $\Delta \delta \alpha=-0,17$ e $\Delta \delta \beta=-0,43$ em $\mathrm{CCl}_{4}$ ), uma maior desproteção no caso de $3\left(\Delta \delta \alpha=0,35\right.$ e $\Delta \delta \beta=-0,04$ em $^{-C_{C H C l}}$ e $\Delta \delta \alpha=$ 0,42 e $\Delta \delta \beta=-0,02$ em $\left.\mathrm{CCl}_{4}\right)$ e um comportamento intermediário para o derivado $4\left(\Delta \delta \alpha=-0,18\right.$ e $\Delta \delta \beta=-0,18$ em $\mathrm{CHCl}_{3}$ e $\Delta \delta \alpha=-0,17$ e $\Delta \delta \beta=-0,11$ em $\left.\mathrm{CCl}_{4}\right)$.

A partir dos estudos na região do infravermelho podemos obter informações valiosas sobre o comportamento estrutural de compostos orgânicos em solução. A princípio, quando se confronta os efeitos gerados pelos átomos de flúor nos experimentos de IV e RMN verifica-se claramente que ambos os efeitos, indutivo e mesomérico, influenciam o equilíbrio conformacional das chalconas em estudo.

Os valores encontrados para as bandas $v_{\mathrm{C}=\mathrm{O}}$ nos espectros de infravermelho da chalcona (1) e seus derivados 2-10 (Tabela 5), em $\mathrm{CHCl}_{3}$ e em $\mathrm{CCl}_{4}$, mostram um aumento no número de onda, na seqüência $1<\mathbf{3}<\mathbf{2}<\mathbf{4}$, tanto para a conformação $s$-cis como para a conformação s-trans, indicando dessa forma um aumento no caráter de ligação dupla do grupo carbonila.

Pode-se observar os mesmos efeitos para os derivados 510 quando comparamos os pares de substâncias 5/6, 7/8 e 9/ 10. No entanto, o aumento do número de grupos $\mathrm{CH}_{2}$ nesta classe de substâncias exerce pouca influência na posição de absorção (Tabela 5).

No caso em particular da carbonila, este aumento do caracter de ligação dupla está de acordo com o efeito indutivo retirador de elétrons dos átomos de flúor, o que pode ser comprovado quando comparamos o aumento da frequiência de estiramento no IV para as carbonilas de ésteres $1750 \mathrm{~cm}^{-1} \mathrm{com}$ as de cetonas $1715 \mathrm{~cm}^{-1}$, uma vez que a região do infravermelho pode fornecer informações valiosas sobre a densidade eletrônica $\pi$, ou mais precisamente, acerca da ordem da ligação.

A análise dos efeitos eletrônicos gerados pelos átomos de flúor sobre os experimentos de RMN ${ }^{1} \mathrm{H} \mathrm{e}{ }^{13} \mathrm{C}$ (que especificamente observa a densidade eletrônica sobre os núcleos, ou seja, o ambiente químico gerado pelo somatório dos efeitos indutivo, mesomérico e anisotrópico), demonstra um predomínio do efeito mesomérico doador dos átomos de flúor, que exercem um efeito de blindagem dos carbonos carbonílicos, como podemos constatar pela observação dos deslocamentos químicos das carbonilas de cetonas $(\delta \sim 200)$ e ésteres $(\delta \sim 170)$, como também com os resultados descritos na literatura para $16^{40}$, demonstrando que o efeito mesomérico contribui predominantemente em C-1.

Pode-se então sugerir que a grande deficiência eletrônica sobre os anéis aromáticos penta-substituídos por flúor, como descrito acima, pode estar de alguma forma polarizando, por efeito indutivo e de campo, todo o conjunto da nuvem eletrônica $\pi$ no híbrido de ressonância por ação das duas unidades contendo átomos de flúor (anéis A e/ou B) sobre o grupo
Carga $q(\mathrm{e})$<smiles>Fc1cc(F)c(F)c(F)c1F</smiles>

$\mathrm{C}_{1}=-0,09$

$\mathrm{C}_{2,6}=0,19$

$\mathrm{C}_{3,5}=0,14$

$\mathrm{C}_{4}=0,14$

$\mathrm{F}_{2-6}=-0,15$

carbonila e refletindo, assim, um aumento de característica da ligação dupla como conseqüência do efeito resultante total, e não apenas atuando sobre os centros individualmente ${ }^{41}$. Isto justificaria assim os resultados aparentemente contraditórios entre os dados de IV e RMN.

Quanto aos estudos comparativos por IV para os derivados 1-4 em $\mathrm{CHCl}_{3}$ e $\mathrm{CCl}_{4}$ (Tabela 5), verificou-se que os valores das absorções na região do IV para os compostos 1-4 em $\mathrm{CHCl}_{3}$ apresentam um deslocamento para menores números de onda quando comparados com os valores em $\mathrm{CCl}_{4}$, o que sugere um maior caracter de ligação dupla da carbonila (Tabela 5). Esse comportamento pode ser resultado de uma menor coplanaridade do sistema de elétrons $\pi$ em $\mathrm{CHCl}_{3}$ e, como conseqüência desta menor coplanaridade do sistema conjugado, o equilíbrio conformacional é deslocado no sentido da conformação s-trans mais polar, o que está de acordo com um ambiente mais polar gerado pelo $\mathrm{CHCl}_{3}$.

A fração molar dos confôrmeros $s$-cis/s-trans em cetonas $\alpha, \beta$-insaturadas $\mathrm{RCOCH}=\mathrm{CHR}$ pode ser calculada a partir dos espectros IV através da razão das intensidades integradas I das bandas correspondentes às conformações $s$-cis e $s$-trans.

$$
\mathrm{I}=1 / \mathrm{c} \cdot 1 \int \mathrm{A}(\overline{\mathrm{v}}) \mathrm{d} \overline{\mathrm{v}}
$$

onde: I é a intensidade média ou aparente de uma banda (área integrada da banda); c a concentração medida para o soluto $\left(\mathrm{em} \mathrm{mol} \mathrm{L} \mathrm{L}^{-1}\right) ; 1$ a espessura da cela $(\mathrm{em} \mathrm{cm})$ e $\mathrm{A}(\overline{\mathrm{v}})$ é a absorbância da amostra a um dado número de onda $\overline{\mathrm{v}}$.

Quando o soluto pode existir em duas conformações, a intensidade específica " $i$ " é definida por $\mathrm{i}=\mathrm{I} / \chi$, onde $\chi$ é a fração molar de um determinado confôrmero em equilíbrio. Parece, entretanto, razoável assumir que na série de compostos $\mathbf{2 - 4}$, análogos da chalcona (1), as intensidades específicas $\mathrm{i}_{\mathrm{C}=\mathrm{O}}(s-$ cis) e $\mathrm{i}_{\mathrm{C}=\mathrm{O}}(s$-trans $)$ sejam constantes ${ }^{33,34}$.

Utilizando-se o valor encontrado para a concentração da conformação $s$-cis $(83 \%)$ no equilíbrio conformacional da chalcona (1) e calculada por $\mathrm{RMN}{ }^{1} \mathrm{H}^{22}$, juntamente com as intensidades aparentes I das bandas $\mathrm{v}_{\mathrm{C}=\mathrm{O}}(s$-cis $)$ e $\mathrm{v}_{\mathrm{C}=\mathrm{O}}(s$-trans $)$ calculadas a partir da área das bandas em $\mathrm{CHCl}_{3} \mathrm{e} \mathrm{CCl}_{4}$, foi possível estimar a fração molar dos derivados 2-4 (Tabela 7). Os resultados obtidos através dessa técnica estão em concordância com as interpretações feitas por RMN tanto em solução de $\mathrm{CDCl}_{3}$ como em $\mathrm{CCl}_{4}$.

\section{CONCLUSÃO}

Os argumentos utilizados neste trabalho para justificar os deslocamentos químicos de H-8, H-7, H-2'/H-6', C-8, C-7 e C-9 $(\mathrm{C}=\mathrm{O})$ basearam-se, essencialmente, nos efeitos anisotrópico (H-8, H-7 e H-2'/H-6'), mesomérico e $\gamma$ protetor (C-8 e C-7) sobre o equilíbrio envolvendo as conformações $s$-cis e s-trans das chalconas. Assim, esta argumentação contém diferenças em relação à descrita na literatura, que leva em consideração somente os efeitos indutivo, mesomérico e indutivo $\pi$ nas atribuições dos deslocamentos químicos para a maioria dos derivados de sistemas carbonílicos $\alpha, \beta$-insaturados. 
Tabela 7. Dados de concentração (\%) para os confôrmeros s-cis e s-trans no equilíbrio conformacional dos compostos $\mathbf{1}$ e $\mathbf{3}$ em $\mathrm{CHCl}_{3}$ e dos compostos $\mathbf{1 - 4}$ em $\mathrm{CCl}_{4}$.

\begin{tabular}{|c|c|c|c|c|c|c|c|c|}
\hline \multirow{2}{*}{$\begin{array}{l}\text { Composto } \\
\text { Conformação }\end{array}$} & \multicolumn{2}{|c|}{1} & \multicolumn{2}{|c|}{2} & \multicolumn{2}{|c|}{3} & \multicolumn{2}{|c|}{4} \\
\hline & $s$-cis & $s$-trans & $s$-cis & $s$-trans & $s$-cis & $s$-trans & $s$-cis & $s$-trans \\
\hline $\mathrm{CHCl}_{3}$ & $83^{\mathrm{a}}$ & $17^{\mathrm{a}}$ & $\mathrm{c}$ & $\mathrm{c}$ & $89,6^{\mathrm{b}}$ & $10,4^{\mathrm{b}}$ & $\mathrm{c}$ & $\mathrm{c}$ \\
\hline $\mathrm{CCl}_{4}$ & $88,7^{b}$ & $11,3^{\mathrm{b}}$ & $67,6^{b}$ & $32,4^{\mathrm{b}}$ & $92,1^{\mathrm{b}}$ & $7,9^{\mathrm{b}}$ & $83,4^{\mathrm{b}}$ & $16,6^{\mathrm{b}}$ \\
\hline
\end{tabular}

${ }^{\mathrm{a}}$ Valores das porcentagens calculados por $\mathrm{RMN}^{22} .{ }^{\mathrm{b}}$ Valores para as percentagens calculados a partir dos pesos das áreas das bandas de absorção na região do infravermelho, tomando como referência os valores calculados por IV para os compostos $\mathbf{1}$ e 3 em $\mathrm{CHCl}_{3}$ e que foram calculados a partir dos resultados de $\mathrm{RMN}{ }^{1} \mathrm{H}$ para o composto (1). ${ }^{\mathrm{c}}$ Valores não calculados devido à superposição das bandas $\mathrm{C}=\mathrm{O}$ e $\mathrm{C}=\mathrm{C}$ para as conformações $s$-cis e s-trans..

A introdução de átomos de flúor tanto no anel A quanto no B da chalcona (1) leva a uma mudança no equilíbrio conformacional s-cis/s-trans, como mostrado tanto qualitativa (por RMN) quanto quantitativamente (por IV), não acarretando nenhuma modificação na configuração $E$ das cetonas estudadas 1-4. Assim, os valores encontrados para a percentagem da conformação s-cis para 1-4 em $\mathrm{CCl}_{4}(88,7 ; 67,6 ; 92,1$ e 83,4 $\%$, respectivamente), e para $\mathbf{1}$ e $\mathbf{3}$ em $\mathrm{CHCl} 3$ (83,0 e 89,6\%, respectivamente), com base no valor calculado por $\mathrm{RMN}{ }^{1} \mathrm{H}$ $(83,0 \%)$ e descrito na literatura para chalcona (1), nos levam a concluir que a substituição por átomos de flúor no anel A tem como conseqüência um aumento na percentagem da conformação $s$-cis no equilíbrio $s$-cis/s-trans, ao passo que a substituição por átomos de flúor no anel B acarreta uma diminuição na percentagem de conformação s-cis no equilíbrio. Este mesmo efeito dos átomos de flúor foi verificado quando se utilizou $\mathrm{CHCl}_{3}$ ou $\mathrm{CCl}_{4}$ como solvente, como observado na comparação dos valores obtidos para 2 e $\mathbf{4}$ com o de chalcona (1). Um segundo efeito é verificado na concentração do equilíbrio conformacional (um aumento da forma $s$-cis), que ocorre quando se muda para solvente menos polar no caso em particular de $\mathrm{CHCl}_{3}$ para $\mathrm{CCl}_{4}$.

Para 5-10, dados de RMN e de IV nos levam a concluir que, em todos os casos, estas cetonas se apresentam em uma configuração $E$ e conformação $s$-cis.

\section{AGRADECIMENTOS}

Os autores agradecem ao CNPq, CAPES e PADCT/FINEP os auxílios e bolsas concedidos.

\section{REFERÊNCIAS}

1. Carey, F. A.; Johnson, S. R.; Advanced Organic Chemistry - Part A, 3rd ed., Plenum Press: New York, 1992. pp 99-100.

2. Huckel, E. Z.; Phys. 1932, 76, 628.

3. Aston, J. G.; Szasz, G.; Woolley, H. W.; Brickwedde, F. G.; J. Chem. Phys. 1946, 14, 67.

4. Bock, C. W.; George, P.; Trachtman, M.; Zanger, M.; J. Chem. Soc. Perkin Trans. 2 1979, 26.

5. Almenninger, A.; Bastiansen, O.; Traetteburg, M.; Acta Chem. Scand. 1958, 12, 1221.

6. Aten, C. F.; Hedberg, L.; Hedberg, K.; J. Am. Chem. Soc. 1968, 90, 2463.

7. Bock, C. W.; George, P.; Tractman, M.; Theor. Chim. Acta. 1984, 64, 293.

8. Furakawa, Y.; Takenchi, H.; Harada, I.; Tasume, M.; Bull. Chem. Soc. Jpn. 1983, 56, 392.

9. Squillacote, M. E.; Sheridan, R. S.; Chapaman, O. L.; Anet, F. A. L.; J. Am. Chem. Soc. 1979, 101, 3657.

10. Squillacote, M. E.; Sheridan, R. S.; Chapaman, O. L.; Anet, F. A. L.; J. Am. Chem. Soc. 1985, 107, 6842.

11. Fisher, J. J.; Michl, L.; J. Am. Chem. Soc. 1987, 109, 1056.

12. Mui, P. W.; Grunwald, E.; J. Am. Chem. Soc. 1982 , 104, 6562 .
13. Breulet, J.; Lee, T. J.; Schaefer III, H. F.; J. Am. Chem. Soc. 1984, 106, 6250.

14. Feller, D.; Davidson, E. R.; Theor. Chim. Acta 1985, $68,57$.

15. Bock, C. W.; George, P.; Trachtman, M.; Theor. Chim. Acta 1984, 64, 193.

16. Wiberg, K. B.; Rosenberg, R. E.; J. Am. Chem. Soc. 1990, $112,1509$.

17. Tai, J. C.; Allinger, N. L.; J. Am. Chem. Soc. 1976, 98, 7928.

18. Arnold, B. R.; Balaji, V.; Michl, J.; J. Am. Chem. Soc. 1990, 112,1812.

19. Wynberg A.; DeGroot, A.; Davies, D. W.; Tetrahedron Lett. 1963, 1083.

20. Roth, W. R.; Lennartz, H. W.; Doering, W. E.; Dolbier, JR, W. R.; Schmidhauser, J. C.; J. Am. Chem. Soc. 1980, $110,1883$.

21. Brouwer, A. M.; Cornelisse, J.; Jacobs, H. J. C.; Tetrahedron 1987, 43, 435.

22. Montaudo, G.; Librando, V.; Caccamese, S.; Maravigna, P.; J. Am. Chem. Soc. 1973, 95, 6365.

23. Devaquet, A. J. P.; Townshend, R. E.; Hehre, W. J.; J. Am. Chem. Soc. 1976, 98, 4068.

24. Dantas T. N. C.; Machado M. I. L.; Braz-Filho R.; Craveiro A. A.; Rev. Latino Amer. Quím. 1984, 15, 25.

25. Lewis, F. D.; Quilen, S. L.; Hale, P. D.; Oxman, J. D.; J. Am. Chem.Soc. 1988, 110, 1261.

26. Eliel, E. L.; Allinger, N. L.; Top. Stereochem. 1969, 5, 167.

27. Turro, N. J. in Modern Molecular Photochemistry, 2nd ed, Benjamin Cummings: Menlo Park, 1978.

28. Kohler, E. P.; Chadwell, H. M.; in Org. Synth. Coll., John Wiley: New York, 1932, Vol I, pp 78.

29. Rabinovich, D.; J. Chem. Soc. (B) 1970, 11.

30. Kipping, F. S.; J. Chem. Soc. 1894, 65, 480.

31. Rapson, W. S.; Shuttleworth, R. G.; J. Chem. Soc. 1940, 636.

32. Günther, H. NMR Spectroscopy, $2^{\text {nd }}$. ed., John Wiley and Sons: New York, 1994.

33. Beinvenue, A.; J Am. Chem. Soc. 1973, 95, 22.

34. Dabrowski, J.; Tenger, M.; Bull. Chem. Soc. Jpn 1975, $48,1310$.

35. Deppmeier, B. J.; Driessen, A. J.; Hehre, A. J.; Hume, T. T.; Johnson, J. A; Klunzinger, P. E.; Lou, L.; Yu, J. Wavefunction, Inc., Irvine, EUA.

36. Solcaniova, E.; Toma, S.; Gronowitz, S.; Org. Mag. Reson. 1976, 8, 439.

37. Solcaniova, E.; Org. Mag. Reson. 1980, 14, 138.

38. Breitmaier, E.; Voelter, W. Carbon-13 NMR Spectroscopy: High-Resolution Methods and Applications in Organic Chemistry and Biochemistry, $3^{\text {rd }}$ ed., VCH: Weinheim, 1987.

39. Jerome, F. R.; Servis, K. L.; J. Am. Chem. Soc. 1972, 94, 5896.

40. Davis, D. W.; Shirley, A.; Thomas, T. D.; J. Am. Chem. Soc. 1972, 94, 6565 .

41. Abraham, R. J.; Angioloni, S.; Edgar, M.; Sancassan, F.; J. Chem. Soc. Perkin Trans. 2 1997, 41. 